CAsE 2.-The patient, aged six years, was well nourished but listless, and did not run about at play but preferred to sit still. Diarrhoea was occasionally suffered from. There was a band of pigmentation round the neck. The posterior folds of the axillæ were also slightly pigmented and there were a well-marked band of pigmentation round the waist and a slight darkening behind the left popliteal space, but there was no involvement of the mucous membrane. There was no other abnormality of note. The blood pressure as measured by the Riva-Rocci instrument showed a maximum systolic pressure of 105 millimetres of mercury.

CASE 3.-The patient, aged three and a half years, was poorly nourished and very listless. Pigmentation involved the neck very slightly and was fairly well marked round the waist, but was not present in other situations. The maximum systolic pressure was 98 millimetres of mercury. The blood showed no abnormality. The eldest sister, aged 17 years, showed no abnormality.

From a study of the three cases, it can hardly be doubted that at any rate Case 1 was a true case of Addison's disease. The prostration, the asthenia, the distribution of the pigmentation, the low blood pressure, and the occasional vomiting go to make a typical picture of the disease, and the only important negative point in this case (common to the three cases) is the absence of pigmentation of the buccal mucous membrane. The second and third cases, while showing only one of the cardinal signs of Addison's diseasenamely, pigmentation, must with a knowledge of the elder sister's condition be viewed with suspicion. The low blood pressure, especially well marked in Case 3 , is also a point of great significance. To revert to Case 1 , an especially interesting feature is the fact that a positive result was obtained by Calmette's tuberculin reaction as pointing to a possible tuberculous condition of the suprarenals. The lesions found post mortem in the adrenals in Addison's disease are in a large majority of cases tuberculous-namely, fibro-caseous. Thus Rolleston in the Goulstonian lectures in summarising the causes of Addison's disease says: "The first [fibro-caseous due to tuberculosis] is the only common cause of Addison's disease." The absence of any definite signs of tuberculous disease, glandular or respiratory, would point to the possibility of a similar lesion present in the case described. The cases as a whole present three main features of interest : (1) the age of the patients ; (2) the family character of the disease; and (3) the low blood pressure in each case. How rare the condition is in children under 13 years of age has been shown, as mentioned by the statistics of Felberbaum and Fruchthandler.

The family character, if we accept Cases 2 and 3 as true cases of Addison's disease, is even more rare, for the only published record is that given by Fleming and Miller, where a mother and four of her children were affected.

Before discussing the third main point of interestnamely, the low blood pressure-it may be well to review briefly some features in regard to the structure and function of the suprarenals as detailed by Rolleston. The suprarenals are composed of (1) the cortex derived from the colomic epithelium in the Wolffian ridge and closely related to the sexual glands ; and (2) the medulla derived from the sympathetic and neuro-ectodermal in origin. As regards the cortex, our imperfeet knowledge of its functions points to the following inferences: (a) that it is related to growth and development; (b) that it has the power of neutralising poisons; and $(c)$ that it is in some way concerned with the internal secretion of the medulla. The function of the medulla is briefly to produce an internal secretion which raises blood pressure by acting on the terminals of the sympathetic.

In the light of this knowledge it is significant to note that the main symptoms in Case 1 are (a) pigmentary changes, (b) asthenia and prostration, and (c) low blood pressure, while in Cases 2 and 3 the pigmentary changes are not so marked but the blood pressure is low. A study of these facts seems to suggest that in cases similar to Case 1 both cortex and medulla are affected, while in those similar to Cases 2 and 3 the medulla is primarily affected and the cortex only slightly so. This view of the cases is in accordance with a hypothesis formed by Dr. Gibson in his address to Guy's Hospital Physical Society in 1907. He says : "It may be supposed that Addison's disease may result in part from inadequacy of the medullary portion leading to asthenia and hypopiesis by loss of adrenalin and by failure of the cortex to maintain certain as yet unknown, but probably important, functions particularly associated with pigmentary excretion and toxin destruction. On this view there may be a most variable assemblage of symptoms, according as the one or the other portion of the suprarenal body is mainly affected." It is impossible to dogmatise from three cases, but as a pure speculation this hypothesis is interesting and the cases recorded appear to add weight to it.

It would be interesting to know in what part of the supra. renal gland the tuberculous lesion begins in Addison's disease-namely, whether the cortex or the medulla is first affected and whether the course of the symptoms is affected differently according as to where the lesion commences. So far as I am aware no definite observations on this point occur in literature, the reason probably being that by the time the cases reach the post-mortem table the lesions involve the whole gland.

The value of a knowledge of the blood pressure in the disease has been emphasised recently, and Cases 2 and 3 , if accepted as Addison's disease, would appear to show that it may be possible to still further differentiate types of the disease and to diagnose a case as such when the pigmentary changes are slight or absent. Stursberg has recently published cases in which the diagnosis of Addison's disease has been made with an almost entire absence of pigmentation and Cases 2 and 3 with low blood pressure, general weakness, and slight gastric and intestinal symptoms may well be associated with the characteristic lesions of the disease.

Bibliography.-Rolleston: Brit. Med. Jour., 1895; The Laycet, Sept. 28th, 1907, p. 875. Stursberg: Munchener Medicinische Wochenschrift, 1907. Gibson: Guy's Hospital Gazette, 1907. Felberbaum and Fruchthandler: New York Medical Journal, 1907 Fleming and

Edinburgh.

\section{INJURY TO THE SEMILUNAR CARTILAGES OF THE KNEE.}

\section{BY RUTHERFORD MORISON, F.R.C.S. ENG. AND} EDIN.,

LECTCRER ON STRGERY, UNIVERSITY OF DURHA M COLLEGE OF MEDICINE, AND SENIOR SURGEON, ROYAL VICTORIA INFIRMARY, NEWCASTLE-ON-TYNE.

SINCE William Hey more than a century ago published ${ }^{1}$ an essay on "Internal Derangement of the Knee-joint," surgery has advanced so materially that knowledge of its pathology through operations on the living has now cleared up many things that were then matters of opinion only. The kneejoint has not escaped this advance, but it is because I think it is possible to make a further small addition to our definite knowledge that I am induced to offer these notes based upon 75 cases operated upon by me from January, 1900, to September, 1908 , on injury to the semilunar cartilages.

It appears to be assumed by authorities that the pathology of these cases is mainly a displacement of the semilunar cartilage, due to a relaxation of the coronary ligaments, but the cases I have seen and operated upon prove that this is wrong, that the injury is a fracture of the cartilage and that displacement without fracture, if it occurs at all, is a very rare event. Of the 75 cases 27 were split longitudinally, 14 were split and detached circumferentially, 8 were split transversely across, 6 were split both transversely and longitudinally, 5 were reported loose, and 5 were reported rupture only. Of 10 the pathology is not recorded. (See illustrations, Figs. 1 to 6 .)

It is of some importance to note that all of the cases reported loose were done in the period when I was unable through an imperfect incision to make a satisfactory examination of the joint, and that during the last two years, since the fact that fracture of the cartilage was the true pathology occurred to me, no case of my own or of my colleagues has been seen to refute this view. On a recent occasion I would have missed the fracture had I not been convinced that it could be found if searched for ; and found it was, but so far back in the joint that it could not be seen eren 
Fig. 1.

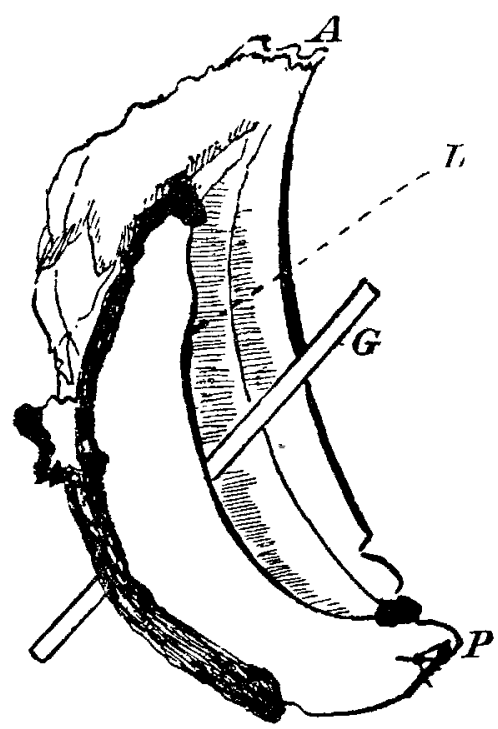

Right internal semilunar cartilage showing longitudinal rupture. A, Anterior end. $\rightarrow$, Glass rod. $\mathbf{L}$, Line of strata separated longitudinally, P. Posterior end.

FIG. 2.

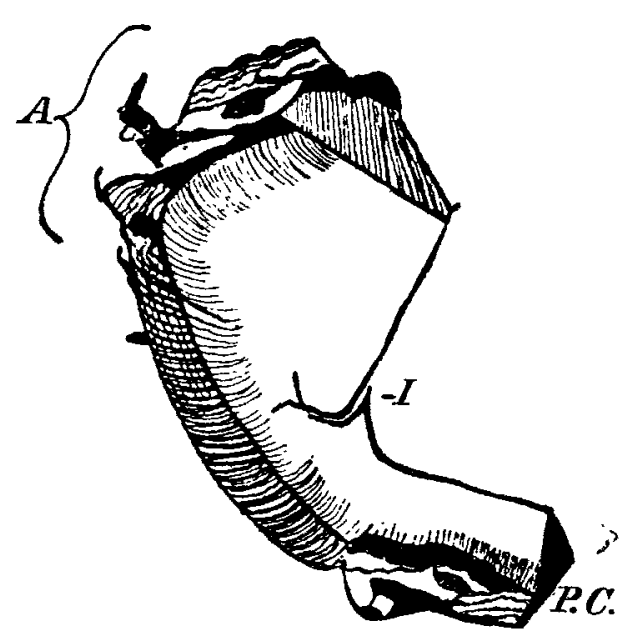

Right internal semilunar cartilage. A, Anterior end detached circumferentially. I, Inner border thinned out between bone ends, with transverse rupture. P.c., Posterior cut end.

FIG. 3.

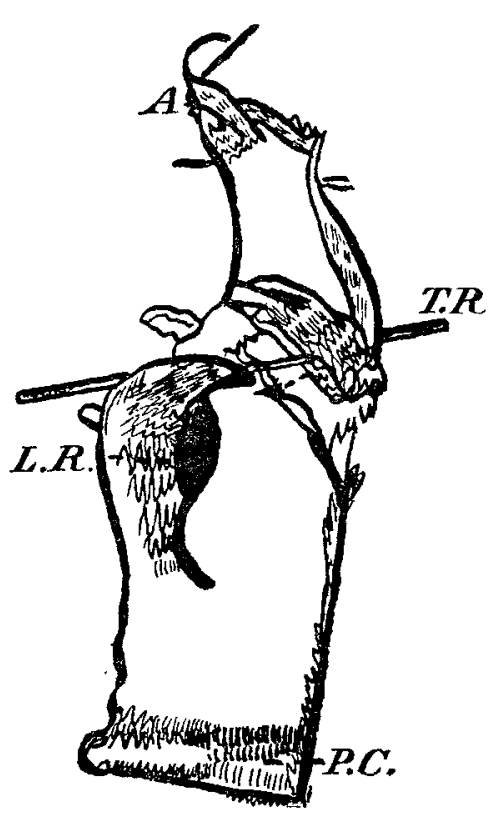

Right internal semilunar cartilage. Under surface. A. Anterior end. 'r.R., Transverse rupture. r. R., Longitudinal rupture. P.C., Posterior cut end.
FIG. 4.

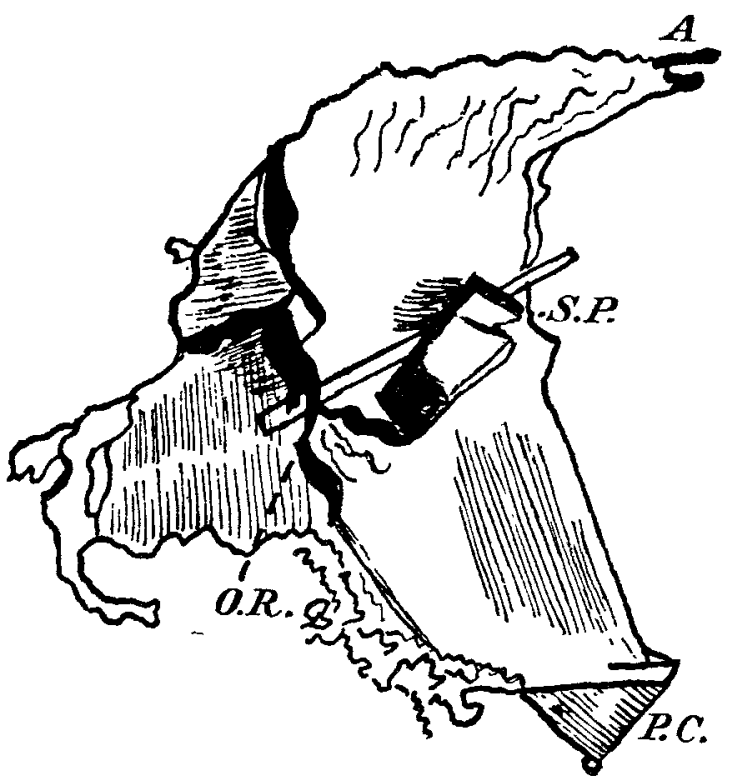

Left internal semilunar cartilage. A, Anterior end. S.P., Split portion. O.R., Old rupture. P.C., Posterior cut end.

FIG. 5.

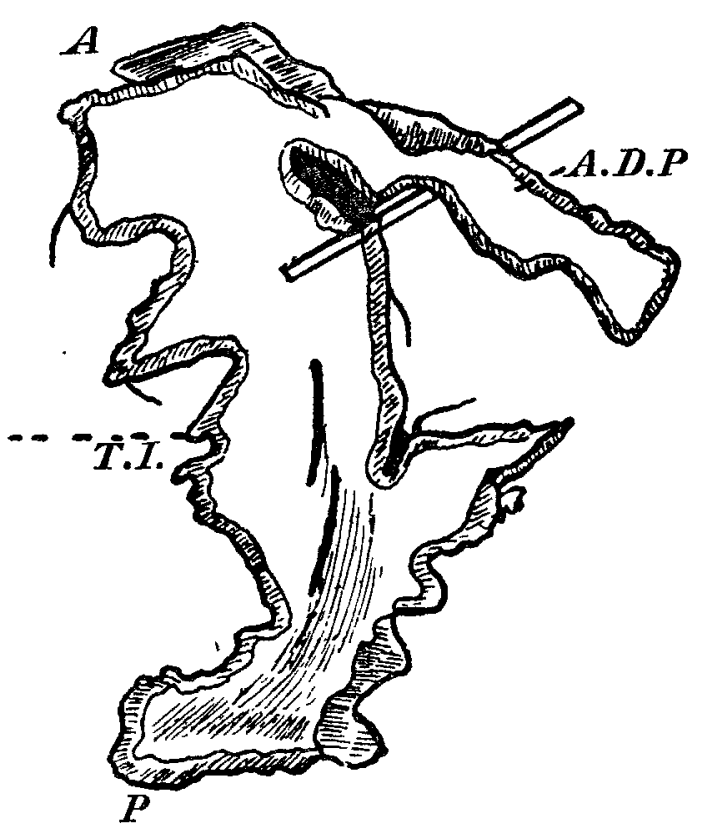

Left internal semilunar cartilage. A, Anterior end. A.D.P., Anterior detached portion. T.I., Thickened inner edge. P., Posterior end

FIG. 6.

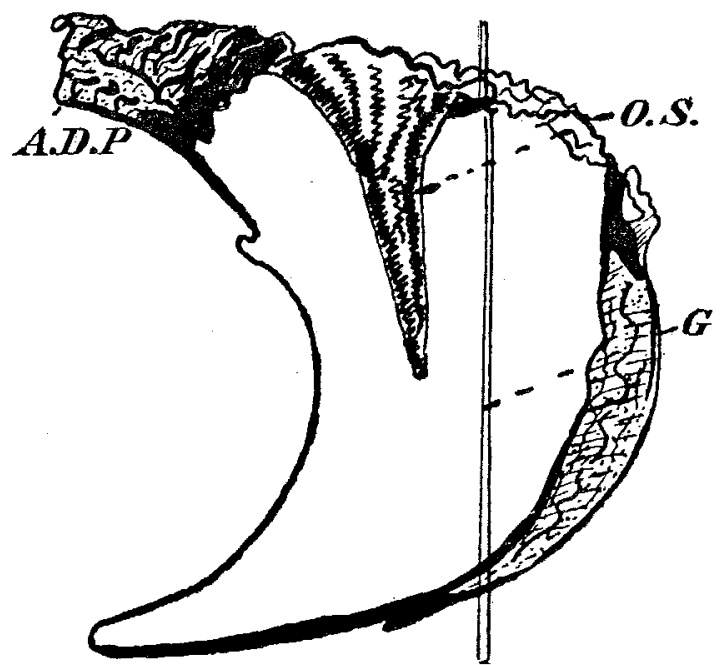

Right internal semilunar cartilage. Under surface. A.D.P., Anterior detached portion. 0.S., Healed old scar. G, Glass rod. 
through the large incision I employed till the joint was fully flexed and the cartilage was forcibly drawn forward. The chief point I want to make is that the cartilage is loose because it is fractured and that this has considerable practical importance. This accident is a common one in miners who work largely with their knees flexed and in awkward positions and usually follows a twist of the flexed knee. Of 100 cases in 98 the internal and in two the external semilunar cartilage are fractured. The injury is attended by very severe pain, sometimes sufficiently serious to cause the strongest man to drop as if he had been shot, and is followed by inability to extend the joint fully and swelling of it. Beyond this history, a twist of the knee followed by a sickening pain and two physical signs: (1) inability to extend the joint fully, and (2) swelling of the joint by fluid in it, seldom anything further is discovered.

In the majority of cases additional testimony is offered to the effect that reduction of the displacement with immediate relief had been brought about after certain movements, such as free flexion, with perhaps external rotation and quick extension, but that some trilling twist at a later date reproduced the symptoms and signs, and that each recurrence seemed to make the next more easy. In the early stages, at least, except when the cartilage is displaced, the joint feels quite well, but later the joint becomes creaky and in a fair percentage of cases osteo-arthritic changes follow and gradually increase till the leg becomes seriously incapacitated. In rare cases a natural cure follows many "attacks." This is probably brought about by fraying out the detached portion till it is destroyed. There appears to be little difference in the liability of the joints to this accident; what there is shows a slight preference for the right, for in my 75 cases 37 were right-sided (36 internal and 1 external), 30 were left-sided ( 29 internal and 1 external), 2 were both knees, and in 6 no record was made. In $89^{\circ}$ cases during the same period from my wards 43 were right-sided, 33 were left-sided, 2 were both knees, and in 11 no record was made.

Diagnosis. - Palpation of the joint rarely, but very rarely, discovers a portion of cartilage moving more freely than normal at the side of the joint affected. Always when the inner side is involved pressure on the anterior edge of the internal lateral ligament elicits tenderness, but this is not a typical sign as it occurs whenever anything inflammatory has occurred in the joint, and is as certainly found as is McBurney's point in appendicitis. The diagnosis is based upon the history of injury, the locking of the joint against full extension and swelling of the joint following the attack. The only conditions it can be confounded with are those in which a solid resisting body falls between the articular surfaces of the femur and tibia and these are to be found in loose cartilages or attached pedunculated cartilaginous growths. So far as I have observed in these cases the patient has always discovered for himself the cause of the trouble and is willing to find and can usually demonstrate it, a thing it is well to let him do. He may experience considerable difficulty in discovering the offender but the surgeon has still more, and when he thinks he has got it the lump may easily slip back into one of the recesses of the joint and disappear, hence the appropriate German name of joint mouse.

Ireatment.-The first thing to be done is to move the displaced cartilage from its false position as soon as possible if this can be accomplished. Flexing the joint to the full extent and then extending it may produce the desired result. If not rotatory movements should be made, in the fully flexed position before extension is performed. If this also fails these attempts should be repeated under an anæsthetic. Immediate relief follows the reduction which is often accompanied by a snap, full extension of the knee is at once possible, and all feeling of tension previously present leaves the hamstring muscles. All the stories that have been published as to the value of this or that special mancuvre for reduction are based upon a wrong pathology and no particular manipulation is likely to succeed if the simple ones described fail. If reduction is successfully accomplished, and this is to be known by the facts that complete and painless extension can be effected, the knee should be fixed by effective splints. For this purpose I prefer two lateral poroplastic splints, strengthened by an iron rib extending from the perineum to the sole of the foot on the inside, and from the trochanter to the sole of the foot on the outside, and fixed with straps or bandages. These are to be worn for six weeks, and with these and a patten on the foot of the sound side and crutches it is allowable for the patient to take exercise. The object of this prolonged rest is to allow of repair of the fractured cartilage, and some f our specimens show that this can be satisfactorily accomplished. For six months at least violent exercise should be voided and the patient should learn to walk with the foot traiglit, or, better still, with the toes slightly inverted. Displacement of the inner cartilage requires that the toes should be everted and a slight injury in this position will reproduce it. For the same reason bicycling is a good exercise because in pedalling the foot is kept straight. If the displacement cannot be reduced the cartilage may either be excised or the knee may be left to see if spontaneous righting occurs, as it not infrequently does. Till painless full extension can be performed splints are of no use and probably diminish the chance of replacement. If the displacement recurs, and especially if it does so after slight provocation, a radical cure is unlikely to follow any measure but excision of the cartilage. A variety of knee supports and trusses have been invented and if the patient prefers the wearing of such a support to the risks of an operation a suitable one can be obtained. For a man whose livelibood depends on his physical capacity and activity such an apparatus is useless - the only thing for him is to face the operation. The danger of this must not be minimised; the operation is many times more serious than any of the ordinary abdominal operations. Surgeons do not, as a rule, publish their misfortunes, but I know of calamities, loss of life, loss of the limb, suppuration of the joint with all its attendant and succeeding horrors, that have happened to skilful and careful surgeons. This has made me cavtious in abandoning methods which have stood the test of time, and in addition to the use of sterile gloves, instruments, towels, and dressings I interfere as little with the joint surfaces as possible, rarely using anything but instruments in the joint, using strong antiseptics ( 1 to 1000 corrosive lotion or 1 in 20 carbolic lotion) for everything not in actual contact with the wound, and weak antiseptics (1 in 10,000) as a final washing for everything else. Surgeons now realise that scientific asepsis of a wound with onr present methods is an impossibility, and in advocating antiseptics it consoles me to remember that a small quantity of antiseptic introduced into a culture medium along with an

\section{FIG. 7.}

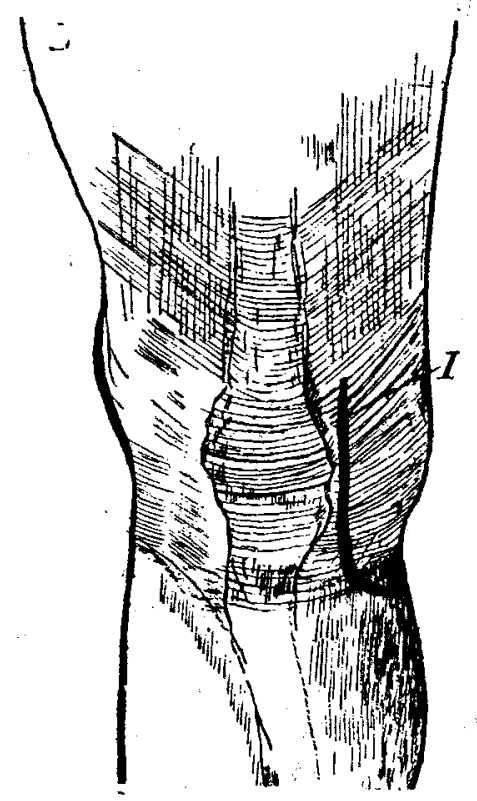

Incision for operation ( $\mathrm{r}$ ).

organism is likely to interfere with its growth, and that even such a feeble antiseptic as boric acid will in infinitesimal quantity delay fermentative changes in milk. Notwithstanding every precaution a large percentage of cases in which the knee-joint has been opened offer evidence of some sepsis. They have severe pain, some rise in temperature, local and general, swelling of the joint, an enlargement of the saphenous glands, and some of them show the red streaks of infected lymphatics. Some ingenious surgical friends suggest that this is due to the irritation of antiseptics, but that is not--considering the very small amount that can get into the wound and the weakness of it and our 
past experience with strong antiseptics in wounds-my own view. The skin over the joint is prepared the night before operation in the usual manner, and again on the table with corrosive lotion once previous to operation.

Operation.-A tourniquet of broad strong indiarubber bandage is applied high on the thigh. After numerous trials without and with the tourniquet I have concluded in favour of this. The knee is flexed and an incision made, as in Fig. 7, through the skin only. The knife with which this incision was made is now rejected as unfit for further use and with a clean knife an incision is made through the extensor aponeurosis from the upper part of the skin incision straight over the head of the tibia. In simple straightforward cases this incision suffices; in more difficult ones the incision is curved backwards over the inner part of the headof the tibia, but must never divide the ligamentous struc tures $^{2}$ on the inner side of the joint beyond the skin incision. A free division of the ligaments here frequently results in a joint capable of some lateral movement, and consequently of a limb incapacitated for any sort of work, a fact which has only been impressed upon me since I have had to examine many miners for the compensation authorities. On dividing the strong extensor aponeurosis a thick pad of tough fat is exposed. This can be drawn forwards between two clip forceps and divided in the line of the previous incision until the joint is opened at some depth. As soon as air gains admission to the synovial cavity the incision can be readily extended to the whole length of the preceding one. With suitable retractors the joint cavity can now be examined, and a blunt hook under the free edge of the cartilage shows whether it is unduly loose and aids the search. If the incision is found to be too small it is extended backwards along the head of the tibia in the joint. Through this incision the joint can be fully explored during first full flexion and then full extension, and if no lesion of the cartilage is discovered further search must be made for the cause of offence. If the cartilage is at fault the crack in it will already have been discovered and the cartilage is to be removed. My general plan is to divide its anterior attachment first; then, holding this in clip forceps, separate it by cutting the coronary attachment back beyond the middle line; then, dividing the cartilage transversely, remove its anterior two-thirds. More than this is not necessary and adds to the difficulties and dangers of the operation. After removal of the cartilage the wound is closed first by a continuous suture of catgut through the extensor aponeurosis, then by a continuous catgut suture fortified by a few interrupted silkworm-gut sutures through the skin. Separate closure of the synovial membrane is unnecessary. In a single case, that of a youth with displaced external semilunar cartilage, on whom I had operated, a small hernia on the outer side of the joint followed the operation. He had used the joint as soon as the wound healed and this may have had to do with the result, but it troubled him so little that he was unaware of its presence till I pointed it out. The wound is dressed with gauze wrung out of 1 in 1000 corrosive spirit lotion, abundant cotton-wool, and a firm bandage. When the dressing is completed, but not till then, the tourniquet is removed whilst the limb is elevated and kept so for a few minutes. Latterly I have discarded all splints and allowed the patient to put the limb in any position he chose. Earlier I kept the knee entirely at rest in efficient splints for an average of three weeks after operation. Now one dressing remains on for three weeks instead. was somewhat of a surprise to me to find in response to our inquiry that the splinted cases were rather better than those treated without splints. The numbers are, of course, too few (given below) and the figures too close to draw any conclusion from and consideration of the fact that individuality, and perhaps compensation in many cases, prolongs the period of abstinence from work after operation has to be taken into account.

After progress.-For a varying time after operation the joint remains weak and occasionally painful when worked, but these symptoms gradually disappear, and after the first year it is the rule for patients to say that the knee is as good as ever it was, and the cases we have inspected, numbering 20 unselected, bear this out fully. We have endeavoured to

2 I purposely avoid saying internal lateral ligament, for this artificial tructure depends on the skilled dissection of the anatomist. All the poneurotic structures are so welded with the ligaments as to be in eparable. obtain the after-history of every case operated upon from 1900 to the end of 1907, not including the last year as these are too recent to form a judgment upon.

Of 58 cases done, 17 cannot be traced; of the 41 returns, 3 cannot work and 38 report themselves able to work. The three cases that are unable to work have been seen and all had extensive osteo-arthritis in the knee-joint, two having had it well marked previous to the operation.

All 38 successful cases were written to and 30 of these replied.

Patients sent out with Splints.

J. S., aged 58 years, began work 4 weeks after operation.

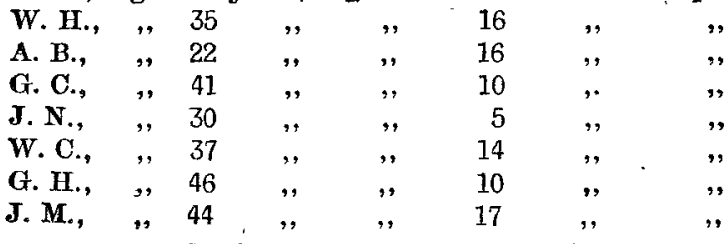

Patients sent out without Splints.

W. C., aged 35 years, began work 24 weeks after operation.

A. $T$.

J. M.,,$\quad 39$

G. T., , 58

G. W., " " 39

W. C., ,, 40

J. McC., , 25

B. C., , 51

M. S., ", 27

J. P.; $\quad$, 52

J. C., , , 32

G. P., , , 46

H. H., , " 20

J. M., , , 39

J. D., , $\quad 30$

W. O., " 38

W. S., , $\quad 36$

M. P., " $\quad 22$

W.R.,

J. P.,

R. A.,

R. G.,

$"$
$"$
$"$
$"$
$"$
$"$,
$"$,
$"$
$"$
$"$
$"$
$"$,
$"$,
$"$
$"$

\begin{tabular}{|c|c|c|}
\hline 28 & & , \\
\hline 7 & ", & ", \\
\hline 12 & ,', & " \\
\hline 20 & " & , \\
\hline 13 & , & $"$ \\
\hline ה & ", & ", \\
\hline 11 & ," & ", \\
\hline & $"$ & ," \\
\hline 11 & ," & יו \\
\hline 13 & ", & 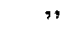 \\
\hline 14 & " & " \\
\hline 12 & '" & $"$ \\
\hline 12 & ", & ," \\
\hline 24 & ", &, \\
\hline 4 & ", & ", \\
\hline 16 & , &, \\
\hline & $"$ & 'ו \\
\hline 16 & ", & ", \\
\hline & , & \\
\hline
\end{tabular}

The average time before, work was commenced by the patients who wore splints is 11 weeks. Those who wore no splints averaged $12 \frac{1}{2}$ weeks.

In connexion with osteo-arthritis it was noted at the operation of several cases that there were thickened hyperæmic synovial membrane, synovial fringes, and some erosion of the cartilages covering the femur or tibia or both. In three of these the disease was arrested by the operation, and the movements of the joints are perfect. In no-single case that we can find was the joint made worse, and no patient was seriously ill after the operation.

For the notes and records of these cases I am indebted to my late house surgeon, Dr. Hamilton Drummond.

Newcastle-on-Tyne.

\section{PANCREATIC SECRETION IN THE TREATMENT OF DIABETES.}

By W. M. CROFTON, M.B., B.CH. R.U.I.

BEFORE describing the case and the treatment adopted $I$ will briefly consider the premises upon which the latter was founded, recapitulating well-known facts so as to make what follows the clearer.

The physiology of the carbohydrates, \&c.-The saliva converts boiled starch and cane sugar into maltose and dextrin. A small amount of inversion also takes place in the stomach. The amylolytic ferment of the pancreas, amylopsin, is the chief agent in this inversion. The maltose and dextrin are then converted into dextrose by a ferment in the intestinal juice, the cells lining the intestine have the same power, and there is also a ferment with a similar function in the blood and tissue juices. The dextrose which is absorbed by the intestinal mucosa is passed into the portal vein which conveys it to the liver cells; these store up a certain amount of it, converting it into glycogen, while the rest passes on to supply the carbohydrate needs of the various tissues ; these also have in a more or less degree the power of converting dextrose into glycogen, forming local 